\title{
Motivación del estudiantado de secundaria en actividades de paisaje sonoro: Un estudio cuasiexperimental en un contexto de vulnerabilidad social
}

\author{
Motivation of Secondary Education Students in Soundscape Activities: \\ A Quasi-Experimental Study in a Context of Social Vulnerability
}

Motivação dos estudantes do ensino médio em atividades de paisagem sonora: Um estudo quase experimental em um contexto de vulnerabilidade social

Rolando Angel-Alvarado

Universidad Pública de Navarra

Pamplona, España rolando.angel.alvarado@gmail.com (D) http://orcid.org/0000-0002-1800-2667

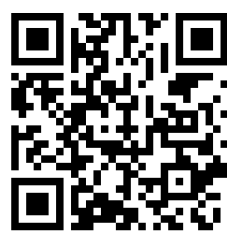

Olga Belletich Universidad Pública de Navarra

Pamplona, España olga.belletich@unavarra.es iD http://orcid.org/0000-0003-2676-6453

Miguel Wilhelmi Universidad Pública de Navarra

Pamplona, España miguelr.wilhelmi@unavarra.es (D) http://orcid.org/0000-0002-6714-7184 
Resumen: La teoría de la autodeterminación (TAD) establece que el comportamiento autodeterminado depende de la satisfacción de tres necesidades psicológicas innatas: autonomía, competencia y afiliación. En este estudio se busca establecer el comportamiento que muestran seis equipos de estudiantes de educación secundaria al desarrollar actividades creativas ligadas al paisaje sonoro, en el marco de la asignatura de música en el sistema educativo chileno. La investigación cuasiexperimental se plantea desde la pedagogía hermenéutica, ya que considera la interacción que existe entre la musicología y la intervención didáctica para interpretar el comportamiento estudiantil desde la TAD. El análisis cualitativo de los datos se sigue de la triangulación de diversos instrumentos, tales como entrevistas focales y técnicas de observación directa. Según los resultados, la mayoría de participantes no muestra comportamientos autodeterminados durante la actividad creativa tras someterse a presiones internas o externas dentro de sus equipos. En conclusión, la autodeterminación depende de la satisfacción de las tres necesidades psicológicas innatas.

Palabras claves: Motivación; educación musical; teoría de la autodeterminación; paisaje sonoro; necesidades psicológicas innatas; actitud laboral.

\begin{abstract}
Self-Determination Theory (SDT) establishes that self-determined behavior depends on the satisfaction of three innate psychological needs: autonomy, competence, and relatedness. In this study, we will establish the behavior that six teams of secondary education students display when carrying out creative activities linked to the soundscape, in the framework of the music subject in Chile's education system. This quasi-experimental study is posed from the hermeneutic pedagogy as it considers the interaction between musicology and didactical intervention for interpreting student behavior in conformity with SDT. The qualitative data analysis entails the triangulation of a variety of tools, such as focal interviews and direct observation techniques. According to the results, most of the participants do not show self-determined behaviors during creative activities as they undergo both internal and external pressures within their teams. In conclusion, self-determination depends on the satisfaction of the three innate psychological needs.
\end{abstract}

Keywords: motivation, music education, self-determination theory, soundscape, innate psychological needs, work attitudes.

Resumo: A teoria da autodeterminação (TAD) afirma que o comportamento autodeterminado depende da satisfação de três necessidades psicológicas inatas: autonomia, competência e afiliação. Neste estudo, procuramos estabelecer o comportamento demonstrado por seis equipes de estudantes do ensino médio ao desenvolver atividades criativas ligadas à paisagem sonora, no contexto do tema da música no sistema educacional chileno. A pesquisa quase experimental propõese desde a pedagogia hermenêutica, já que considera a interação que existe entre a musicologia e a intervenção didática para interpretar o comportamento das pessoas estudantes desde a TAD. Posterior a análise qualitativa dos dados segue a triangulação de vários instrumentos, como entrevistas focais e técnicas de observação direta. Segundo os resultados, a maioria de participantes não mostra comportamentos autodeterminados durante a atividade criativa depois de se submeter a pressões internas ou externas dentro de suas equipes. Em conclusão, a autodeterminação depende da satisfação das três necessidades psicológicas inatas.

Palavras-chave: Motivação; educação musical; teoria da autodeterminação; paisagem sonora; necessidades psicológicas inatas; atitude de trabalho 


\section{Introducción}

La creación de paisajes sonoros depende de la motivación que experimente el sujeto durante la actividad creativa. Por ello, asume relevancia la teoría de la autodeterminación (TAD), porque permite distinguir, en la actitud motivada, dos tipos de motivación: autónoma y controlada (Deci y Ryan, 1980). La motivación autónoma es entendida como la experiencia que involucra el sentido de voluntad y la capacidad de elección, mientras que la motivación controlada se comprende como la sensación de sometimiento o subyugación durante la actividad (Gagné y Deci, 2005). Así, las personas que poseen mayor motivación autónoma muestran un comportamiento autodeterminado porque son capaces de regular su conducta en función del placer que la actividad creativa les comporta (Fernet, Guay y Senécal, 2004).

Por consiguiente, las motivaciones autónoma y controlada no son dicotómicas, al contrario, se evidencian simultáneamente durante la actividad (Parker, Jimmieson y Amiot, 2010) porque, por ejemplo, un sujeto creativo puede actuar con sentido de voluntad en labores que demandan el cumplimiento de tareas en plazos prefijados. Por lo tanto, la conducta autodeterminada es facilitada o interrumpida por diversos factores personales y ambientales.

Se establecen tres categorías para definir los tipos motivacionales:

- Desmotivación, definida como la ausencia de motivación hacia una actividad (Gagné et al., 2014). Representa el máximo déficit motivacional, es decir, el límite inferior de la motivación caída (Legault, Green-Demers y Pelletier, 2006).

- Motivación extrínseca, tiene un comportamiento instrumental que provoca una internalización continua (Ryan, Kuhl y Deci, 1997), por lo tanto, a mayor internalización de la motivación extrínseca, mayor autonomía muestra la persona en su comportamiento. Se compone de tres reguladores (Gagné et al., 2014):

- Externa, implica buscar recompensas o evitar castigos.

- Interna, es la regulación de la conducta a través de las fuerzas internas como ego, culpabilidad, vergüenza, etc.

- Identificada, realizar una actividad por identificarse con el valor o significado, aceptándola como acción propia, a pesar de no conllevar una satisfacción inherente, sino un valor instrumental.

- Motivación intrínseca, representa el compromiso en función del placer o satisfacción que deriva de la realización de la actividad, de modo que es afectada por la motivación extrínseca (Salanova, Hontangas y Peiró, 2007).

De acuerdo con la integración orgánica (Ryan, 1995), la TAD sugiere que el ser humano naturalmente se integra dentro de una estructura social dinámica, de modo que el proceso de adaptación implica un interés de inserción social, un compromiso por las actividades colectivas 
doi: http://dx.doi.org/10.15359/ree.23-2.18

URL: http://www.una.ac.cr/educare

CORREO: educare@una.cr

y el desarrollo de acciones interpersonales en la relativa unidad (Deci y Ryan, 2000). En este marco, la TAD dispone tres necesidades psicológicas innatas (Deci et al., 2001) que requieren ser apoyadas por la condición ambiental y las relaciones interpersonales para su satisfacción (Ryan y Deci, 2002). Así, se establecen las técnicas claves de asistencia a las necesidades (Silva, Marques y Teixeira, 2014), que definen las condiciones interpersonales y su progreso operacionalmente. Las necesidades y sus asistencias son:

1. Autonomía: Es el deseo de elección y sentimiento de voluntad en las acciones. La asistencia-autonómica comprende la evitación del control mediante métodos autoritarios, propiciar ambientes de respeto por la actividad propia y de otras personas, fomentar la capacidad de elección e incentivar el autorreconocimiento.

2. Competencia: Es el deseo de interactuar de forma eficaz en el contexto. La asistenciacompetencial radica en la oportunidad de entrenamiento guiado y en la estructura de las tareas. Es importante la claridad de expectativas, retos acordes con las habilidades y la retroalimentación constructiva.

3. Afiliación: Es la sensación de conexión con los demás seres. La asistencia-social se forja en la sintonización con el entorno mediante la atención y el conocimiento interpersonal. Cobra relevancia la empatía; el afecto genuino por los otros seres; la dedicación de recursos, si la contingencia lo requiere, y la confianza para estar disponible en caso necesario.

La TAD propone que la motivación autónoma depende de la satisfacción de las tres necesidades psicológicas innatas (Gagné, 2009), ya que los contextos que apoyan todas las necesidades básicas tienden a mejorar la motivación intrínseca y a facilitar la internalización de los reguladores de la motivación extrínseca (Deci y Ryan, 2000). Además, la TAD establece la hipótesis de que la satisfacción de las tres necesidades psicológicas innatas favorece el desempeño en acciones creativas, como también en tareas que demandan resoluciones efectivas (Gagné y Deci, 2005), porque la actividad no se limita al desempeño de la tarea, sino también implica el disfrute de la acción (Deci y Ryan, 2000).

En la educación musical, la TAD se está comenzando a observar en el proceso de enseñanzaaprendizaje con el afán de crear ambientes sociales que potencien el comportamiento autodeterminado en actividades didáctico-musicales (Evans, 2015).

En aquella línea, este estudio tiene la finalidad de establecer el comportamiento que experimenta el estudiantado de Primer Año Medio (catorce a quince años de edad) al desarrollar actividades creativas de paisaje sonoro, para lo que se establecieron los siguientes objetivos específicos: a) observar la dificultad que representa la actividad creativa para el estudiantado que conforma la muestra; b) determinar el medio de presentación del producto creativo que prevalece en la muestra y;c) analizar la motivación que demuestran los sujetos durante la situación didáctica. 
Para alcanzar estos objetivos, se ha diseñado un experimento en el que el estudiantado asiste a sesiones de inducción sobre el paisaje sonoro, donde, por un lado, el cuerpo estudiantil debe establecer consensos con su docente para efectuar el proyecto creativo (en particular, sobre la constitución de los equipos de trabajo según sus gustos e intereses) y donde, por otro lado, el estudiantado debe exhibir su producto musical en las condiciones que el equipo determine internamente. Estas predeterminaciones aseguran las condiciones ambientales para observar comportamientos autodeterminados en el estudiantado, conforme las restricciones naturales que representa una actividad escolar ligada a la evaluación sumativa (Sicherl, Kordeš y Holcar, 2017).

\section{Método}

Esta investigación cuasiexperimental se desarrolla desde la pedagogía hermenéutica establecida para la educación musical (Ángel-Alvarado, Wilhelmi y Belletich, 2017) porque existe una interacción sistemática y continua entre la investigación y la acción concreta en el sistema de enseñanza.

La didáctica filosófica de la música (Georgii-Hemming y Lilliedahl, 2014) permite comprender el circulo hermenéutico. Allí, el paisaje sonoro, como elemento musicológico, interacciona con las cuatro dimensiones de la didáctica de la música: Didáctica básica, etnodidáctica, desafío-didáctico y didáctica antropológica. Por lo tanto, la interacción propicia la construcción de conocimiento musicológico para el campo de la educación musical, en el marco que el paisaje sonoro delimita.

Este estudio se centra en la didáctica antropológica porque lo importante es establecer el comportamiento autodeterminado (o no) estudiantil en un proyecto colectivo de paisaje sonoro, en el marco de la asignatura de música establecida en el sistema educativo chileno. Así, la TAD cobra relevancia para la interpretación de la didáctica antropológica, ya que hace factible la aproximación hacia el tipo motivacional del cuerpo estudiantil.

\section{Participantes}

El estudio se ha realizado en una escuela mixta de dependencia particular subvencionada localizada en la zona central de Chile. El grupo lo constituyen treinta estudiantes de educación secundaria que cursan el nivel de primer año medio (catorce a quince años de edad). La escuela, según los registros públicos del Ministerio de Educación de Chile, tiene características similares a otros establecimientos del país, ya que atiende adolescentes provenientes de familias de clase media-baja, con un cuarenta por ciento en condiciones de vulnerabilidad social.

En el marco de la educación musical, la escuela comienza a impartir la asignatura desde séptimo año básico (doce a trece años de edad), esto quiere decir que la muestra está cursando su tercer año académico de formación musical. Además, la mayoría de participantes integra la 
doi: http://dx.doi.org/10.15359/ree.23-2.18

URL: http://www.una.ac.cr/educare

CORREO: educare@una.cr

banda escolar encargada de musicalizar los desfiles oficiales, de modo que posee un bagaje musical que supera las competencias que establece el currículo institucional.

En el grupo muestral se registra que seis estudiantes se han incorporado a la institución durante el curso de primer año medio y que la totalidad proviene de otros colegios cercanos a la escuela participante. De ellos, cuatro adolescentes informan haber cursado educación musical durante su proceso de escolarización previo y dos comunican que nunca han participado en clases de educación musical. Toda la muestra participante ha cursado una unidad de nivelación musical al inicio del año escolar, por lo que se decide no descartar a este par de estudiantes.

\section{Instrumentos}

Se han construido estrategias de recolección de datos pertinentes a los tipos motivacionales de la TAD. Estas técnicas fueron validadas por un panel experto de educación musical, pedagogía y psicología educativa, como también por el profesor especialista y la dirección curricular del centro educativo. Posteriormente, durante el mismo año, las técnicas se validaron ante una muestra piloto conformada por estudiantes de primer año medio de la misma escuela.

La investigación está circunscrita en un proyecto sonoro-musical, de modo que se utilizan las siguientes técnicas:

- Entrevista focal:Durante el proceso creativo, se conversa con cada equipo para establecer la representación colectiva que tienen del proyecto (por ejemplo, se pregunta a los grupos "¿es importante la realización del proyecto para su desarrollo personal?"); la opinión de sus propias competencias musicales (p.e., ¿piensan que pueden mostrar un alto desempeño durante la ejecución del paisaje sonoro?); como también, ahondar en su capacidad de gestión para elaborar un proyecto creativo-musical (p. e., ¿qué tareas cumplirá cada quien a lo largo del proyecto?). Esta pauta permite determinar el grado de dificultad que el proyecto representa al equipo de trabajo.

- Registro anecdótico 1: Durante el proceso creativo, se invita a los equipos a exponer sus proyectos al resto de la clase, una instancia para recibir retroalimentaciones de los pares. En esta situación didáctica, se registra el comportamiento que muestran los equipos mientras comunican su proyecto (p.e., ¿su disertación es elocuente?) y la tolerancia durante la retroalimentación de compañeros y compañeras (p.e., ¿asumen con respeto las críticas de sus pares?). Esta técnica permite establecer la actitud que muestra cada sujeto hacia el trabajo, permitiendo establecer los patrones actitudinales comunes de cada equipo.

- Evaluación objetiva, rúbrica: El producto de paisaje sonoro se evalúa según criterios estético-musicales, considerándose esencialmente la riqueza rítmica (p.e., ¿es polirítmica la performance?), la propuesta timbrística (p.e., ¿denota una exploración sonora exhaustiva la propuesta timbrística?) y la recreación de un paisaje sonoro (p.e., ¿recrea 
el equipo un paisaje sonoro?). Con estos criterios, es posible determinar la calidad del producto sonoro-musical que el equipo exhibe.

- Evaluación de desempeño: Se evalúa el trabajo de cada integrante del equipo, para determinar si ha tenido una participación activa durante la realización del proyecto. En primera instancia, se corrobora que el sujeto tuvo un rol activo durante la performance (p.e., ¿participa activamente durante toda la performance?) y, posteriormente, se conversa con el equipo para recopilar información laboral de cada integrante (p.e., ¿cumple cada integrante las tareas asignadas?). Esta técnica permite establecer el desempeño del equipo y sus integrantes, a lo largo del proyecto.

- Registro anecdótico 2: Los equipos pueden presentar su performance de la manera que decidan, por lo tanto, se registra el formato de presentación empleado (p.e., ¿qué formato utiliza el equipo para presentar su trabajo?). Esta técnica hace factible registrar el formato de exhibición del producto sonoro.

Las técnicas señaladas responden a la dimensión didáctica antropológica, porque los datos recogidos se interpretan según las bases teóricas de la TAD (Figura 1).

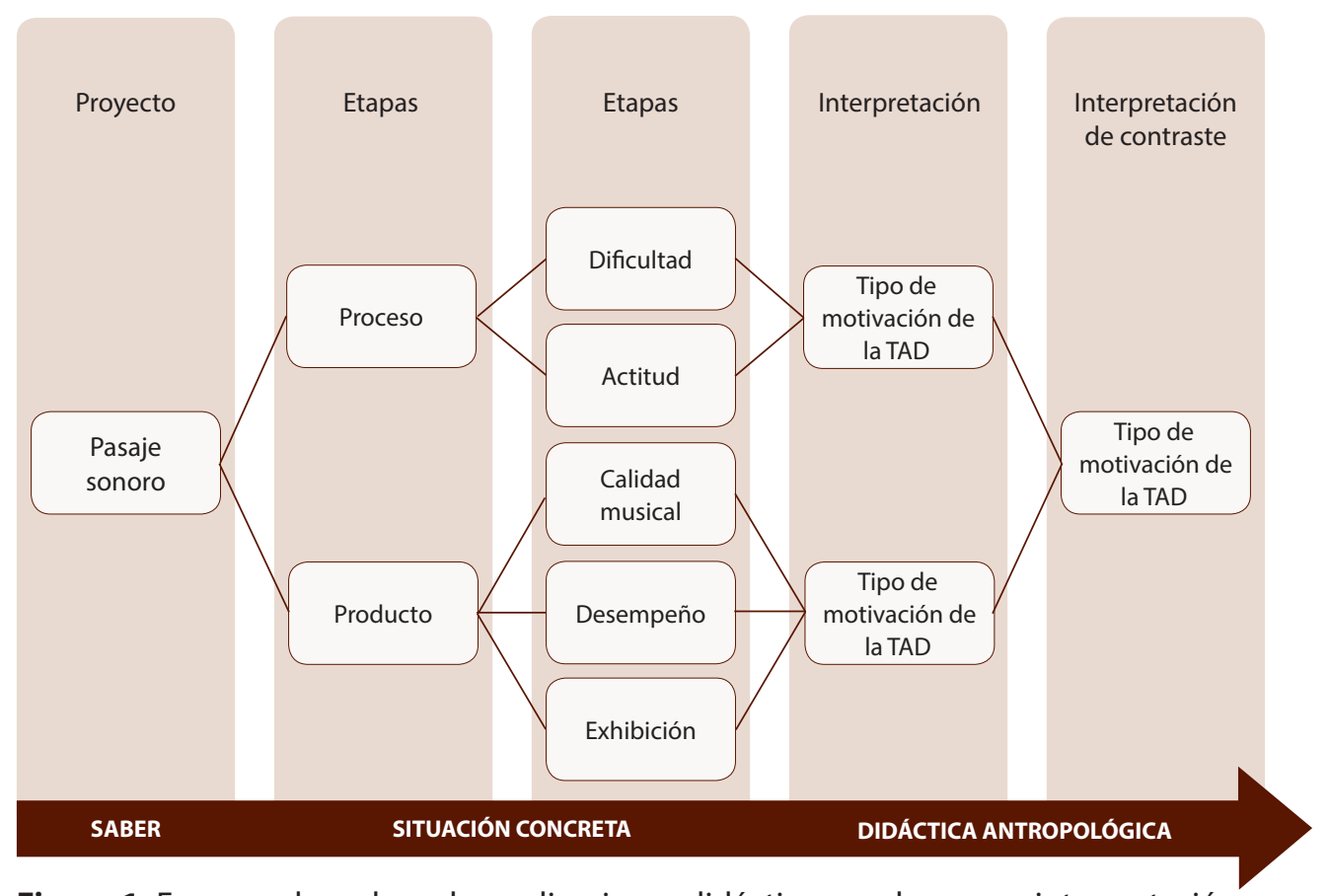

Figura 1: Esquema basado en las realizaciones didácticas en clases y su interpretación. Nota: Elaboración propia. 
doi: http://dx.doi.org/10.15359/ree.23-2.18

URL: http://www.una.ac.cr/educare

CORREO: educare@una.cr

\section{Procedimientos}

Se programan cuatro sesiones de noventa minutos cada una, desde un enfoque prácticocrítico (Aróstegui, 2014).

En la primera sesión, se abordan las cualidades del sonido y algunos fenómenos acústicos (eco y reverberación), desde la discriminación auditiva de los fenómenos sonoros y repertorios musicales, la imitación de los eventos sonoros al utilizar el cuerpo humano y las posibilidades sonoras que brindan los dispositivos tecnológicos.

En la segunda sesión, se reflexiona sobre la importancia que tuvo para el ser humano primitivo el acto de imitar la naturaleza mediante los sonidos y los movimientos corporales. Posteriormente, se aprecian audiovisualmente agrupaciones sonoro-musicales irreverentes como Stomp o Mayumana, quienes rompen los estándares tradicionales al utilizar fuentes sonoras que no responden a instrumentos musicales convencionales, dando pie a un debate sobre la experiencia estética. Finalmente, se establecen consensos sobre las normas para desarrollar el proyecto creativo de paisaje sonoro, estableciendo que los equipos de trabajo deben estar constituidos por entre cuatro y ocho integrantes, teniendo que garantizarse la participación activa de la totalidad a lo largo de la representación artística. Se cuenta con dos semanas de proceso creativo, y se fija que al concluir la primera semana cada equipo se somete a una evaluación formativa frente a la clase y al concluir la segunda semana, presenta su producto creativo en el marco de la evaluación sumativa o acreditativa. Se acuerda que los equipos tienen amplia libertad para realizar sus creaciones, pudiendo presentarlas en vivo o mediante video. Solamente se exige que el tiempo de presentación esté dentro del intervalo 2.00-2.30 minutos. Esta limitación busca que el estudiantado sea capaz de comunicar un mensaje sintético, lo más preciso y preclaro posible, sin limitarse a una intervención excesivamente breve o a una acumulación excesiva sin estructura inteligible.

Durante la tercera sesión, se procede a observar la dificultad (alta, aceptable, baja) que representa la situación didáctica para los equipos, como también, las categorías motivacionales. Se realizan entrevistas focales a cada grupo con la intención de recoger información sobre la motivación externa del equipo, ahondando en el clima organizacional interno, la opinión que tienen sobre sus competencias creativo-musicales y el imaginario colectivo que han construido sobre la actividad. Luego, se observan las actitudes (positiva, indiferente, negativa, no aplica) que muestra el estudiantado durante la evaluación formativa frente al resto de la clase, ya que deja en evidencia su grado de autodeterminación en la actividad.

En la cuarta sesión, se constata el medio de presentación que prefieren los distintos equipos y se decide filmar el producto creativo de los equipos que han optado por mostrar su obra en forma presencial, para evaluar los diversos elementos que establecen los instrumentos de evaluación. Todas estas variables permiten observan los tipos motivacionales [TM] que 
muestran los participantes conforme a la TAD: autonomía [AUTO] y controlada [CONTROL]. La ausencia de cualquier TM, se clasifica como desmotivación [DESMOT].

Finalmente, se contrastan los TM obtenidos por cada equipo durante el proceso creativo y el producto sonoro-musical, permitiendo definir el TM que predomina durante el desarrollo de la situación didáctica.

Es conveniente comunicar que, por cuestiones éticas de la actividad investigadora, la identidad del estudiantado participante no se develará mediante ninguna vía y en ningún escenario, puesto que los sujetos son menores de edad. Aún cuando existan consentimientos que autoricen su difusión, el equipo de investigación ha decidido no compartir imágenes con la intención de salvaguardar la identidad del alumnado participante.

\section{Resultados y su discusión}

La muestra se ha dividido en seis equipos (Tabla 1). Esta distribución acontece de forma natural ya que el estudiantado ha montado libremente sus equipos de trabajo.

Tabla 1: Distribución de la muestra en equipos de trabajo

\begin{tabular}{cc}
\hline Equipo & Estudiantes \\
\hline Equipo 1 & 4 \\
Equipo 2 & 4 \\
Equipo 3 & 5 \\
Equipo 4 & 4 \\
Equipo 5 & 6 \\
Equipo 6 & 7 \\
\hline
\end{tabular}

Nota: Elaboración propia.

\section{Proceso creativo del paisaje sonoro}

La situación didáctica representa una dificultad aceptable para tres equipos, porque en todos los casos, la mayoría de sus integrantes piensa poseer las competencias creativo-musicales que la situación didáctica demanda. Además, se observa que alguna persona integrante ha asumido la dirección del proyecto creativo, lo que muestra diversos perfiles de liderazgo. En el caso particular de los equipos 2 y 3, se constatan las técnicas claves de asistencia a las necesidades psicológicas innatas (Silva et al., 2014), ya que fomentan el trabajo participativo, asignan tareas apropiadas a cada integrante y planifican el producto creativo conforme las 
doi: http://dx.doi.org/10.15359/ree.23-2.18

URL: http://www.una.ac.cr/educare

CORREO: educare@una.cr

capacidades del equipo. El equipo 5, por su parte, ha alzado una dirección autoritaria, lo que representa un obstáculo, porque no satisface todas las necesidades psicológicas innatas. Según la $T A D$, en contextos de motivación controlada, las necesidades de competencia y afiliación se pueden satisfacer; sin embargo, las personas no muestran un compromiso óptimo y un bienestar psicológico (Ryan, 1993).

Para los otros tres equipos, la actividad representa una dificultad alta, ya que nadie muestra una actitud proactiva ni asume el liderazgo, por lo tanto, no estructuran tareas, ni establecen metas. Además, su opinión competencial es poco convincente y la representación que han construido sobre el proyecto es negativa, especialmente porque aseveran que la educación musical no tiene ninguna relevancia en sus pretensiones futuras. Conforme a la TAD, estos tres equipos de trabajo se categorizan en la desmotivación al no tener intenciones de desarrollar el proyecto y al caracterizarse por sentimientos de alienación y desamparo (Ryan y Deci, 2002).

Durante la disertación frente al curso, la mayoría de los equipos asume una actitud negativa o indiferente, ya que las exposiciones eran poco amistosas y en especial el equipo 5 , rechaza todas las aportaciones dadas por sus pares. Particularmente el equipo 2 , decide no participar en la actividad sin expresar razones, de modo que la decisión responde a reguladores internos de la motivación extrínseca (Gagné et al., 2014) como la vergüenza. La excepción es el equipo 3, que muestra una actitud entusiasta, ya que genera expectativas por su trabajo; por lo tanto, son los únicos capaces de regular su conducta en función del placer que la actividad creativa les comporta (Fernet et al., 2004).

Al tomar en cuenta la dificultad y la actitud (Tabla 2), se determina que el TM que prevalece durante el proceso creativo es la desmotivación, seguida por la autonomía y finalmente, la controlada.

Tabla 2: Tipo motivacional del equipo en el proceso creativo, según DIFICULTAD y ACTITUD

\begin{tabular}{cllllll}
\hline Equipos & Equipo 1 & Equipo 2 & Equipo 3 & Equipo 4 & Equipo 5 & Equipo 6 \\
\hline Dificultad & Alta & Aceptable & Aceptable & Alta & Aceptable & Alta \\
Actitud & Indiferente & No aplica & Positiva & Indiferente & Negativa & Negativa \\
\hline TM & DESMOT & AUTO & AUTO & DESMOT & CONTROL & DESMOT \\
\hline
\end{tabular}

Nota: Elaboración propia.

Los TM observados no representan un resultado final, ya que serán contrastados con los resultados del producto musical para establecer el TM que muestran los equipos a lo largo de la situación didáctica. 


\section{Producto creativo del paisaje sonoro}

En la Tabla 3, se observa que los equipos 2 y 3 son los únicos en recrear un paisaje sonoro, mostrando una variedad timbrística y una riqueza rítmica. Además, los equipos que respetan la condición composicional son los únicos en fomentar la motivación autónoma, porque satisfacen las tres necesidades psicológicas innatas (Deci et al., 2001). Satisfacen la autonomía al fomentar el respeto y el trabajo participativo (Su y Reeve, 2011) porque permiten que cada integrante asuma responsabilidades conforme a sus posibilidades. Asimismo, satisfacen la competencia al estructurar las tareas y definir expectativas acordes con las capacidades de sus integrantes (Haerens et al., 2013). Por último, favorecen la afiliación al establecer un clima de confianza, donde existe sintonía entre integrantes (Van den Broeck, Vansteenkiste, De Witte, Soenens y Lens, 2010). Todo esto representa una aproximación a la confirmación de la hipótesis establecida por la TAD: la satisfacción de las necesidades psicológicas innatas favorece el desempeño en actividades creativas, como también en tareas que requieren resoluciones efectivas (Gagné y Deci, 2005).

En cuanto al desempeño, se observa que el equipo 5 divide su trabajo audiovisual en cinco escenas independientes, dando preferencia de participación a quienes dirigen. Así, el resto de integrantes no aparece en todas las escenas del video. La evidencia empírica de la TAD demuestra que las personas altamente eficaces no satisfacen óptimamente sus necesidades psicológicas innatas, cuando persiguen (y logran) metas extrínsecas atribuibles a cuestiones culturales (Ryan y Deci, 2000). Esta evidencia se replica en el equipo 5, ya que la dirección está dispuesta a postergar el bienestar del equipo, con tal de obtener un buen resultado académico en la evaluación sumativa.

Todos los equipos deciden exponer sus productos a través del formato audiovisual (Tabla 3), por diferentes razones. El equipo 2 incluye fenómenos acústicos (eco y reverberación) durante la edición del video. El equipo 3 ha ambientado su paisaje sonoro en una ferretería. El equipo 5 ha secuenciado su presentación en cinco escenas independientes. Finalmente, en los productos de los equipos 1, 4 y 6, no se constatan justificaciones técnicas para publicar un video, por lo tanto, actúan en concordancia con su desmotivación (Legault et al., 2006), porque aceptan someterse al control, con un producto musical que deja en evidencia su elaboración procrastina.

Tabla 3: Tipo motivacional del equipo en el producto creativo, según CALIDAD, DESEMPEÑO y EXHIBICIÓN

\begin{tabular}{ccccccc}
\hline \multicolumn{1}{c}{ Equipos } & Equipo 1 & Equipo 2 & Equipo 3 & Equipo 4 & Equipo 5 & Equipo 6 \\
\hline Calidad & Baja & Aceptable & Aceptable & Baja & Aceptable & Baja \\
Desempeño & Bajo & Aceptable & Aceptable & Bajo & Bajo & Bajo \\
Exhibición & Video & Video & Video & Video & Video & Video \\
\hline TM & CONTROL & AUTO & AUTO & CONTROL & CONTROL & CONTROL \\
\hline
\end{tabular}

Nota: Elaboración propia. 
doi: http://dx.doi.org/10.15359/ree.23-2.18

URL: http://www.una.ac.cr/educare

CORREO: educare@una.cr

Como información adicional, el equipo 6 no respeta los tiempos acordados, ya que durante la jornada se niega a participar en la exhibición de productos creativos, y solicita al final de la sesión una extensión al plazo de entrega. Tras cumplir el nuevo acuerdo, el equipo 6 categoriza en la motivación controlada, ya que actúa motivado por los reguladores externos e internos.

La influencia cultural cobra valor en el TM de los equipos, lo que implica que el personal docente y el contexto inciden en las metas externas que los equipos establecen. La TAD toma en cuenta el componente ecológico, al señalar que la obtención de metas influenciadas culturalmente no satisface plenamente las necesidades psicológicas innatas, a pesar de mostrar un rendimiento altamente eficaz (Ryan y Deci, 2000). En otras palabras, la TAD entiende la obtención de metas externas como una conducta ligada a la motivación controlada; por lo tanto, el hecho que la mayoría de los equipos califique en el TM de control, da cuenta de la existencia de una cultura focalizada en la obtención de resultados académicos.

\section{Contrastación de tipos motivacionales}

En la Tabla 4, se observa que los equipos mantienen el TM calificado durante el producto creativo.

Tabla 4: Contrastación del tipo motivacional del equipo en las distintas etapas de la situación didáctica

\begin{tabular}{lcccccc}
\hline Equipos & Equipo 1 & Equipo 2 & Equipo 3 & Equipo 4 & Equipo 5 & Equipo 6 \\
\hline TM (proceso) & DESMOT & AUTO & AUTO & DESMOT & CONTROL & DESMOT \\
TM (producto) & CONTROL & AUTO & AUTO & CONTROL & CONTROL & CONTROL \\
\hline TM & CONTROL & AUTO & AUTO & CONTROL & CONTROL & CONTROL \\
\hline
\end{tabular}

Nota: Elaboración propia.

En el caso de los equipos 1, 4 y 6, categorizan en la motivación controlada porque presentan el producto creativo a la evaluación sumativa, a pesar de mostrar desmotivación durante toda la situación didáctica. Estos equipos están focalizados en la obtención de un resultado académico que solo les permita aprobar, porque su producto musical da cuenta de una realización procrastina.

Los equipos 2 y 3 categorizan en la motivación autónoma porque muestran comportamientos autodeterminados a lo largo de la situación didáctica. Además, son los únicos que recrean un paisaje sonoro y que satisfacen las tres necesidades psicológicas innatas. Por lo tanto, se confirma la hipótesis de la TAD porque la satisfacción de todas las necesidades favorece el desempeño en actividades creativas y en tareas que demandan resoluciones efectivas. 
En cuanto al equipo 5, se enmarca en la motivación controlada porque su dirección ha mostrado conductas de presión y sometimiento durante toda la situación didáctica, sin satisfacer todas las necesidades psicológicas innatas. Todo esto ha sido gatillado por metas externas, como la obtención de un excelente resultado académico en el producto creativomusical durante la evaluación sumativa.

Estas evidencias empíricas hacen factible la utilización de la TAD en el proceso de enseñanza-aprendizaje de la educación musical, porque permite interpretar la interacción escolar, para potenciar el comportamiento autodeterminado en actividades didáctico-musicales (Evans, 2015).

La práctica pedagógico musical necesita a la TAD para mejorar su desempeño, porque la interacción didáctica al adecuarse al saber, al contexto, al personal docente y al alumnado (Burnard, 2016; Sicherl et al., 2017), le atribuye a cada docente un grado de responsabilidad por el comportamiento autodeterminado (o no) que muestra el estudiante. Si bien, este estudio no indaga en la interacción del personal docente con los equipos, ni en la del contexto, la tendencia hacia la motivación controlada hace latente la relevancia de la influencia cultural (Ryan y Deci, 2000). Por ello, es conveniente que en estudios futuros se consideren las variables ecológicas del contexto de aula, como también los contratos didácticos docente-estudiante.

\section{Análisis desde teorías complementarias a la TAD}

La teoría cognitivo-experiencial (Epstein, 1998) considera dos sistemas de aprendizaje paralelos e interactivos que determinan el comportamiento del ser humano: el sistema experiencial y el sistema racional. En su interacción, el sistema experiencial facilita la adquisición y desarrollo de aprendizajes significativos en el sistema racional. En este marco de interacción continua, solo los equipos 2 y 3 adquieren aprendizajes musicológicos significativos, porque instauran dinámicas de interacción social que satisfacen las tres necesidades psicológicas innatas. En cambio, los aprendizajes significativos (si es que los hay) del equipo 5 responden a cuestiones sociales, porque han experimentado una serie de conflictos interpersonales. Los equipos restantes difícilmente han adquirido aprendizajes significativos.

En este sentido, los contextos educativos construyen imaginarios según sus modelos educativo-participativos, los que influyen en las relaciones interpersonales y en otras variables contextuales como las asignaturas y los métodos de enseñanza (Belletich y Wilhelmi, 2012). Así en el contexto de estudio, la cultura escolar se focaliza en los resultados académicos, antes que en el proceso de desarrollo personal del estudiantado. De hecho, la mayoría de los equipos cumplen con su responsabilidad estudiantil porque necesitan aprobar el curso, lo que implica un sometimiento a presiones externas, es decir, motivación controlada. 
doi: http://dx.doi.org/10.15359/ree.23-2.18

URL: http://www.una.ac.cr/educare

CORREO: educare@una.cr

\section{Conclusiones}

El éxito de la situación didáctica depende, en gran medida, de la conducta autodeterminada de liderazgo que asuma al menos un sujeto integrante, pues las técnicas claves de asistencia a las necesidades psicológicas innatas son unos factores determinantes. Se observa que tan solo los equipos que satisfacen la autonomía, la competencia y la afiliación, son los que muestran capacidad de gestión y cumplen con la recreación de un paisaje sonoro. Por lo tanto, se confirma la hipótesis de la TAD, ya que la satisfacción de las tres necesidades psicológicas innatas favorece el desempeño en actividades creativas y en tareas que demandan resoluciones efectivas. Con todo esto, se asume que los equipos que muestran comportamientos autodeterminados son los únicos en adquirir aprendizajes significativos de orden musicológico.

Los equipos restantes no muestran comportamientos autodeterminados, porque no satisfacen todas las necesidades psicológicas innatas, como tampoco cumplen la tarea efectiva de recrear un paisaje sonoro. En este escenario, es poco probable que los sujetos adquieran aprendizajes musicológicos significativos. La influencia cultural que los satura desde el contexto escolar hace que se focalicen en la obtención de metas externas como el rendimiento académico, impidiendo la satisfacción óptima de las tres necesidades psicológicas innatas y el disfrute por la actividad educativa.

En conclusión, los equipos de estudiantes muestran comportamientos autodeterminados (motivación autónoma) y no autodeterminados (motivación controlada). Por una parte, los equipos autodeterminados consiguen cumplir la actividad creativa satisfactoriamente, porque disfrutan tanto de la situación didáctica, que adquiere un valor personal que supera el imaginario construido sobre la evaluación sumativa o acreditativa. Por otra parte, los equipos no autodeterminados no son capaces de cumplir las estipulaciones técnicas establecidas para la recreación del paisaje sonoro, porque desarrollan la situación didáctica en función de la obtención de una buena calificación académica, sin disfrutar la tarea didáctica centrada en la creatividad.

La mayoría de los equipos califica en el comportamiento no autodeterminado, lo que denota la influencia cultural del contexto, ya sea promovido por la figura docente, el proyecto educativo del centro o ambas. En esta línea, es necesario profundizar el análisis del proceso de enseñanza-aprendizaje, donde es fundamental incluir la variable docente, como también, la variable ecológica. Estos elementos hacen posible determinar la influencia cultural que porta el estudiantado.

Igualmente, como medida de corto plazo, es conveniente establecer planes de mejora en el curso observado, para lograr que todo el estudiantado alcance la motivación autónoma. El cuerpo estudiantil se ha distribuido libremente para el proyecto sonoro, por lo tanto, se sugiere no mantener la misma distribución en proyectos futuros, para fomentar el intercambio cultural e incitar la zona del desarrollo próximo del alumnado rezagado en competencias musicales. Quizás 
deba ser la figura docente quien conforme los equipos de trabajo para asegurar la presencia de un liderazgo autodeterminado en cada equipo, y evitar la ejecución de mecanismos pedagógicos que impliquen una imposición en el estudiantado. Es decir, se puede recurrir a dinámicas que involucren el azar o la selección aleatoria durante la construcción de los equipos de trabajo.

De este modo, surgen una serie de implicaciones. Por un lado, desde un punto de vista formativo, es necesario promover el pensamiento creativo en las actividades didácticomusicales, ya que el estudiantado muestra dificultades para emprender proyectos de creación musical. Por otro lado, se precisa promover la implementación de estrategias pedagógicas que reduzcan la práctica estudiantil procrastina, centrando tanto la instrucción como la evaluación en el proceso y en el producto que surge de la didáctica musical. Finalmente, es esencial orientar las situaciones didáctico-musicales hacia el constructivismo social para que cada estudiante pueda compartir experiencias socio-musicales con la comunidad escolar, dejando atrás la pedagogía tradicional centrada en la relación diádica estudiante-repertorio, ya que ha quedado en evidencia que, al abordar actividades creativas de paisaje sonoro, el estudiantado tiene dificultades para emprender proyectos grupales con sus pares.

Con todo esto, se confirma que la TAD es pertinente para investigar el comportamiento estudiantil en actividades musicales ligadas al paisaje sonoro, porque permite interpretar comportamientos que se suscitan durante la acción concreta en la clase de música, aportando conocimiento empírico relevante para mejorar la práctica pedagógica en el campo de la educación musical.

\section{Referencias}

Ángel-Alvarado, R., Wilhelmi, M. R. y Belletich, O. (Agosto, 2017). Design-based research for Music Education: Didactical engineering. Trabajo presentado en la European Conference Educational Research, Copenhague. Recuperado de http://www.eera-ecer.de/ecerprogrammes/conference/22/contribution/40777/

Aróstegui, J. L. (2014). Fundamentos del currículo para la educación musical. En J. L. Aróstegui (Ed.), La música en educación primaria: Manual de formación del profesorado (pp. 19-44). Madrid: Dairea.

Belletich, O. y Wilhelmi, M. R. (2012). Estrategia de enseñanza-aprendizaje por acción razonada. Transmisión y adquisición del modelo educativo y de los criterios pedagógicos en educación infantil. En P. Miralles y A. B. Mirete (Eds.), La formación del profesorado en educación infantil y educación primaria (pp. 315-322). Murcia: Universidad de Murcia.

Burnard, P. (2016). Problematizing what counts as knowledge and the production of knowledges in music. En E. Georgii-Hemming, P. Burnard y S.-E. Holgersen (Eds.), Professional knowledge in music teacher education (pp. 97-108). Nueva York, NY: Routledge. 
doi: http://dx.doi.org/10.15359/ree.23-2.18

URL: http://www.una.ac.cr/educare

CORREO: educare@una.cr

Deci, E. L. y Ryan, R. M. (1980). Self-determination theory: The iteration of psychophysiology and motivation. Psychophysiology, 17(3), 321.

Deci, E. L. y Ryan, R. M. (2000). The "what" and "why" of goal pursuits: Human needs and the self-determination of behavior. Psychological Inquiry, 11(4), 227-268. doi: http://dx.doi. org/10.1207/S15327965PLI1104 01

Deci, E. L., Ryan, R. M., Gagné, M., Leone, D. R., Usunov, J. y Kornazheva, B. P. (2001). Need satisfaction, motivation, and well-being in the work organizations of a former eastern bloc country: A cross-cultural study of self-determination. Personality and Social Psychology Bulletin, 27(8), 930-942. doi: https://doi.org/10.1177/0146167201278002

Epstein, S. (1998). Constructive thinking: The key to emotional intelligence. Westport: Praeger.

Evans, P. (2015). Self-determination theory: An approach to motivation in music education. Musicae Scientiae, 19(1), 65-83. doi: https://doi.org/10.1177/1029864914568044

Fernet, C., Guay, F. y Senécal, C. (2004). Adjusting to job demands: The role of work selfdetermination and job control in predicting burnout. Journal of vocational behavior, 65(1), 39-56. doi: https://doi.org/10.1016/S0001-8791(03)00098-8

Gagné, M. y Deci, E. L. (2005). Self-determination theory and work motivation. Journal of Organizational Behavior, 26(4), 331-362. doi: https://doi.org/10.1002/job.322

Gagné, M. (2009). A Model of knowledge-sharing motivation. Human Resource Management, 48(4), 571-589. doi: https://doi.org/10.1002/hrm.20298

Gagné, M., Forest, J., Vansteenkiste, M., Crevier-Braud, L., Van den Broeck, Aspeli, A.-K., ... Westbye, C. (2014). The multidimensionnal work motivation scale: Validation evidence in seven languages and nine countries. European Journal of Work and Organizational Psychology, 24(2), 178-196. doi: https://doi.org/10.1080/1359432X.2013.877892

Georgii-Hemming, E. y Lilliedahl, J. (2014). Why "what" matters: On the content dimensions of music didactics. Philosophy of Music Education Review, 22(2), 132-155. doi: https://doi. org/10.2979/philmusieducrevi.22.2.132

Haerens, L., Aelterman, N., Van den Berghe, L., De Meyer, J., Soenens, B. y Vansteenkiste, M. (2013). Observing physical education teachers' need-supportive interactions in classroom setting. Journal of sport \& exercises psychology, 35(1), 3-17. doi: https://doi.org/10.1123/jsep.35.1.3

Legault, L., Green-Demers, I. P. y Pelletier, L. (2006). Why do high school students lack motivation in the classroom? Toward an understanding of academic amotivation and the role of social support. Journal of Educational Psychology, 98(3), 567-582. doi: https://doi. org/10.1037/0022-0663.98.3.567 
Parker, S. L., Jimmieson, N. L. y Amiot, C. E. (2010). Self-determination as a moderator of demands and control: Implications for employee strain and engagement. Journal of Vocational Behavior, 76(1), 52-67. doi: https://doi.org/10.1016/j.jvb.2009.06.010

Ryan, R. (1993). Agency and organization: Intrinsic motivation, autonomy and the self in psychological development. En J. E. Jacobs (Ed.), Nebraska symposium on motivation 1992: Developmental perspectives on motivation (Vol. 40, pp. 1-56). Lincoln: University of Nebraska Press.

Ryan, R. (1995). Psychological needs and the facilitation of integrative processes. Journal of Personality, 63(3), 397-427. doi: https://doi.org/10.1111/j.1467-6494.1995.tb00501.x

Ryan, R. M., Kuhl, J. y Deci, E. L. (1997). Nature and autonomy: An organizational view of social and neurobiological aspects of self-regulation in behavior and development. Development and Psychopathology, 9(4), 701-728. doi: https://doi.org/10.1017/S0954579497001405

Ryan, R. M. y Deci, E. L. (2000). Self-determination theory and the facilitation of intrinsic motivation, social development, and well-Being. American Psychologist, 55(1), 68-78. doi: https://doi.org/10.1037/0003-066X.55.1.68

Ryan, R. M. y Deci, E. L. (2002). An overview of self-determination theory: An organismicdialectical perspective. En E. L. Deci y R. Ryan (Eds.), Handbook of self-determination research (pp. 3-33). Rochester: University of Rochester Press.

Salanova, M., Hontangas, P. y Peiró, J. (2007). Motivación laboral. En J. M. Peiró y F. Prieto (Eds.), Tratado de psicología del trabajo. Vol 1: La actividad laboral en su contexto (pp. 215-249). Madrid: Síntesis.

Sicherl, B., Kordeš, U. y Holcar, A. (2017). Assessment for learning in music education in the Slovenian context - from punishment or reward to support. Music education research, 19(1), 17-28. doi: https://doi.org/10.1080/14613808.2015.1077800

Silva, M., Marques, M. M. y Teixeira, P. J. (2014). Testing theory in practice: The exampled of selfdetermination theory-based interventions. The European Health Psychologist, 16(5), 171-180. Recuperado de http://www.ehps.net/ehp/index.php/contents/article/view/ehp.v16.i5.p171/9

Van den Broeck, A., Vansteenkiste, M., De Witte, H., Soenens, B. y Lens, W. (2010). Capturing autonomy, competence, and relatedness at work: Construction and initial validation of the work-related basic need satisfaction scale. Journal of occupational and organizational psychology, 83(4), 981-1002. doi: https://doi.org/10.1348/096317909X481382

Su, Y.-L. y Reeve, J. (2011). A meta-analysis of the effectiveness of intervention programs designed to support autonomy. Educational psychology review, 23(1), 159-188. doi: https:// doi.org/10.1007/s10648-010-9142-7 